\title{
SIMULATION ANALYSIS OF SYSTEM LIFE WHEN COMPONENT LIVES ARE DETERMINED BY A MARKED POINT PROCESS
}

\author{
SHELDON M. ROSS, ${ }^{*}$ University of Southern California
}

\begin{abstract}
We consider an $r$ component system having an arbitrary binary monotone structure function. We suppose that shocks occur according to a point process and that, independent of what has already occurred, each new shock is one of $r$ different types, with respective probabilities $p_{1}, \ldots, p_{r}$. We further suppose that there are given integers $n_{1}, \ldots, n_{r}$ such that component $i$ fails (and remains failed) when there have been a total of $n_{i}$ type- $i$ shocks. Letting $L$ be the time at which the system fails, we are interested in using simulation to estimate $\mathbb{E}[L], \mathbb{E}\left[L^{2}\right]$, and $\mathbb{P}(L>t)$. We show how to efficiently accomplish this when the point process is (i) a Poisson, (ii) a renewal, and (iii) a Hawkes process.
\end{abstract}

Keywords: Simulation; marked point process; variance reduction; renewal process; conditional expectation; Hawkes process; Poissonization

2010 Mathematics Subject Classification: Primary 91B70; 68U20

\section{Introduction}

Suppose that $r$ components, each of which is either working or failed at any point of time, constitute a system having a binary nondecreasing structure function $\phi$. That is, whether or not the system is working depends solely on which components are working, and if $x_{i}$ is the indicator for the event that component $i$ is working then $\phi\left(x_{1}, \ldots, x_{n}\right)$ is the indicator for the event that the system is working. Suppose that shocks occur according to a point process, that each shock is one of $r$ types, and that independent of what has previously occurred each new shock is type $i$ with probability $p_{i}, \sum_{i=1}^{r} p_{i}=1$. Furthermore, suppose that for specified integers $n_{1}, \ldots, n_{r}$, component $i$ fails (and remains failed) when there have been a total of $n_{i}$ type- $i$ shocks, $i=1, \ldots, r$. Letting $L$ be the time at which the system fails, we are interested in efficiently using simulation to estimate $\mathbb{E}[L], \mathbb{E}\left[L^{2}\right]$, and $\mathbb{P}(L>t)$.

Let $N(t)$ denote the number of shocks by time $t$, and let $S_{n}$ be the time of the $n$th shock. Also, let $N$ be the number of shocks that have occurred at the moment that the system fails; that is, $L=S_{N}$. Because the type of a new shock is independent of what has previously occurred, it follows that $N$ is independent of the process $\{N(t), t \geq 0\}$, and so the conditional distribution of $L$ given $N=n$ is the unconditional distribution of $S_{n}$. Thus, for instance,

$$
\mathbb{P}(L>t \mid N=n)=\mathbb{P}\left(S_{n}>t\right)=\mathbb{P}(N(t)<n)
$$

and

$$
\mathbb{E}[L \mid N=n]=\mathbb{E}\left[S_{n}\right]
$$

Received 20 February 2013; revision received 24 May 2013.

* Postal address: Department of Industrial and Systems Engineering, University of Southern California, Los Angeles, CA 90089, USA. Email address: smross@usc.edu

This material is based upon work supported by, or in part by, the US Army Research Laboratory and the US Army Research Office under contract/grant number W911NF-11-1-0115. 
In Section 2 we consider the multinomial stopping time random variable $N$. We use Poissonization both in generating such a random variable and in obtaining efficient estimators of $\mathbb{E}[N], \mathbb{E}\left[N^{2}\right]$, and $\mathbb{P}(N>m)$. The estimator of $\mathbb{P}(N>m)$ is a conditional expectation estimator, whereas the estimators of $\mathbb{E}[N]$ and $\mathbb{E}\left[N^{2}\right]$ are linear combinations of a conditional expectation estimator and a second unbiased estimator. In Section 3 we show how to estimate $\mathbb{E}[L], \mathbb{E}\left[L^{2}\right]$, and $\mathbb{P}(L>t)$ in the cases where $N(t), t \geq 0$, is, respectively, a Poisson process, a renewal process, and a Hawkes process. Some concluding remarks are made in Section 4. Throughout, we use the notation $\mathbf{1}\{A\}$ to represent the indicator variable for the event $A$, equal to 1 if $A$ occurs and to 0 otherwise.

\section{Multinomial stopping time $N$}

Consider multinomial trials, where each trial results in one of the outcomes $1, \ldots, r$ with respective probabilities $p_{1}, \ldots, p_{r}$. We say that the quota for type- $i$ events is met when there have been at least $n_{i}$ of them, $i=1,2, \ldots, r$. Let $X_{i}(n)$ equal 1 if the quota for type- $i$ events has not been met when $n$ trials have occurred, and let it equal 0 otherwise. The random variable $N$ is defined by

$$
N=\min \left\{n: \phi\left(X_{1}(n), \ldots, X_{r}(n)\right)=0\right\} .
$$

For instance, if $1 \leq k \leq r$ and

$$
\phi(x)=1\left\{\sum_{i=1}^{r} x_{i} \geq k\right\}
$$

then $N$ is the number of trials until there have been at least $n_{i}$ type- $i$ outcomes for exactly $r-k+1$ of the values $i=1, \ldots, r$. If, for given constants $c_{1}, \ldots, c_{r}, c$,

$$
\phi(x)=\mathbf{1}\left\{\sum_{i=1}^{r}\left(1-x_{i}\right) c_{i}<c\right\}
$$

then we can imagine that a cost $c_{i}$ is incurred whenever there have been $n_{i}$ type- $i$ outcomes and that the process stops when the total incurred cost is at least $c$.

To obtain an efficient way to estimate $\mathbb{E}[N]$, we use the standard Poissonization trick (see [2] or [6]) of supposing that the times at which trials are performed constitute the event times of a Poisson process with rate $\lambda=1$. If we then say that the Poisson event is of type $i$ if the trial outcome is $i$ then, with $N_{i}(t)$ equal to the number of type- $i$ events by time $t$, it follows that the processes $\left\{N_{i}(t), t \geq 0\right\}$ are, for $i=1, \ldots, r$, independent Poisson processes with respective rates $p_{i}$. Hence, if we let $T_{i}$ be the time until there have been a total of $n_{i}$ type- $i$ trial outcomes then $T_{1}, \ldots, T_{r}$ are independent gamma random variables with respective parameters $\left(n_{i}, p_{i}\right)$.

To obtain our estimators, generate the random variables $T_{1}, \ldots, T_{r}$ and order them so that $T_{I_{1}}<T_{I_{2}}<\cdots<T_{I_{r}}$. For a subset $S \subset\{1, \ldots, r\}$, let $x_{i}(S)=\mathbf{1}\{i \notin S\}$ and define

$$
\phi(S)=\phi\left(x_{1}(S), \ldots, x_{r}(S)\right) .
$$

That is, $\phi(S)$ is the indicator of whether a system with structure function $\phi$ works when $S$ is the set of failed components.

Now, define

$$
K=\min \left(j=1, \ldots, r: \phi\left(\left\{I_{1}, \ldots, I_{j}\right\}\right)=0\right)
$$

and set $T=T_{I_{K}}$. Thus, $T$ is the time of trial number $N$. Hence, with $N_{j}=N_{j}(T)$ equal to the 
number of type- $j$ trial outcomes by time $T$, we have

$$
N=\sum_{j=1}^{r} N_{j}=\sum_{j=1}^{r} N_{I_{j}}
$$

Now, for $j<K$, the quota of type- $I_{j}$ outcomes was met at time $T_{I_{j}}$ and the additional number of type- $I_{j}$ outcomes that would have occurred by time $T$ is Poisson distributed with mean $p_{I_{j}}\left(T-T_{I_{j}}\right)$. For $j>K$, the $n_{I_{j}}$ th outcome of type $I_{j}$ occurred at time $T_{I_{j}}>T$. As the joint distribution of the times of the first $n_{I_{j}}-1$ type- $I_{j}$ outcomes is that of independent uniformly distributed random variables on the interval $\left(0, T_{I_{j}}\right)$, it follows that the conditional distribution of $N_{I_{j}}$ given $\boldsymbol{T}=\left(T_{1}, \ldots, T_{r}\right)$ is, for $j>K$, that of a binomial random variable with parameters $n_{I_{j}}-1, T / T_{I_{j}}$. As the number of type- $I_{K}$ trial outcomes by time $T$ is $n_{I_{K}}$, and as all of the preceding binomial and Poisson random variables are conditionally independent given $\boldsymbol{T}$, it follows that

$$
N \mid \boldsymbol{T}={ }_{d} n(\boldsymbol{T})+X(\boldsymbol{T})+\sum_{j=K+1}^{r} B_{j}(\boldsymbol{T}),
$$

where $n(\boldsymbol{T})=\sum_{j=1}^{K} n_{I_{j}}$ and $X(\boldsymbol{T})$ and $B_{K+1}(\boldsymbol{T}), \ldots, B_{r}(\boldsymbol{T})$ are conditionally independent given $\boldsymbol{T}$. Here $X(\boldsymbol{T})$ is Poisson distributed with mean $\sum_{j=1}^{K-1} p_{I_{j}}\left(T-T_{I_{j}}\right)$, and $B_{j}(\boldsymbol{T}), j>K$, is binomially distributed with parameters $\left(n_{I_{j}}-1, T / T_{I_{j}}\right)$.

\subsection{Estimating $\mathbb{E}[N]$}

It follows from (1) that

$$
\mathbb{E}[N \mid \boldsymbol{T}]=\sum_{j=1}^{K} n_{I_{j}}+\sum_{j=1}^{K-1} p_{I_{j}}\left(T-T_{I_{j}}\right)+\sum_{j=K+1}^{r}\left(n_{I_{j}}-1\right) \frac{T}{T_{I_{j}}},
$$

which yields an unbiased estimator of $\mathbb{E}[N]$. A second unbiased estimator is obtained by noting that

$$
T=\sum_{j=1}^{N} Y_{j}
$$

where $Y_{1}, Y_{2}, \ldots$ are the interarrival times of the Poisson process. Because these interarrival times are independent of the successive event types, and thus of $N$, we see that

$$
\mathbb{E}[T \mid N]=N \mathbb{E}\left[Y_{j}\right]=N,
$$

showing that $T$ is also an unbiased estimator of $\mathbb{E}[N]$. If we had to choose between the two unbiased estimators of $\mathbb{E}[N]$ then $\mathbb{E}[N \mid \boldsymbol{T}]$ would be the choice since, by the Rao-Blackwell theorem,

$$
\operatorname{var}(T) \geq \operatorname{var}(\mathbb{E}[T \mid N])=\operatorname{var}(N) \geq \operatorname{var}(\mathbb{E}[N \mid \boldsymbol{T}]) .
$$

However, there is no need to choose, as we can do better by taking an estimator of the form $\alpha \mathbb{E}[N \mid \boldsymbol{T}]+(1-\alpha) T$, which is equivalent to using the estimator $\mathbb{E}[N \mid \boldsymbol{T}]$ along with the zero-mean control variate $\mathbb{E}[N \mid \boldsymbol{T}]-T$. The estimated best value of $\alpha$ would be determined from the simulation (see Section 9.2 of [7]).

Remark. An approach to compute the mean of a stopping time of a sequence of multinomial trials by using Dirichlet integrals was given in [8]. 
Example. Suppose $r=2, n_{1}=n_{2}=1, p_{1}=p_{2}=\frac{1}{2}$, and that the system is parallel and thus fails when there has been at least one outcome of each type. As $N$ is distributed as 1 plus a geometric with parameter $\frac{1}{2}$, it follows that $\operatorname{var}(N)=2$. Now, $\mathbb{E}[N \mid \boldsymbol{T}]=2+\frac{1}{2}\left(T_{(2)}-T_{(1)}\right)$, where $T_{(1)}, T_{(2)}$ are the ordered values of the exponential random variables $T_{1}, T_{2}$. As $T_{(2)}-T_{(1)}$ is exponential with rate $\frac{1}{2}$,

$$
\operatorname{var}(\mathbb{E}[N \mid \boldsymbol{T}])=1 .
$$

Because $T=T_{(2)}$, using the estimator $\mathbb{E}[N \mid \boldsymbol{T}]$ along with the zero-mean control variate

$$
Y \equiv \mathbb{E}[N \mid \boldsymbol{T}]-T=2-\frac{1}{2}\left(T_{(1)}+T_{(2)}\right)
$$

yields, when the best constant $c$ (obtained from the simulation) is used,

$$
\frac{\operatorname{var}(\mathbb{E}[N \mid \boldsymbol{T}]+c Y)}{\operatorname{var}(\mathbb{E}[N \mid \boldsymbol{T}])}=1-\operatorname{corr}^{2}(\mathbb{E}[N \mid \boldsymbol{T}], Y) .
$$

Because $T_{(2)}$ is equal to $T_{(1)}$ plus an independent exponential with rate $\frac{1}{2}$, we have

$$
\operatorname{var}\left(T_{(2)}\right)=1+4=5, \quad \operatorname{cov}\left(T_{(1)}, T_{(2)}\right)=\operatorname{var}\left(T_{(1)}\right)=1 .
$$

Hence, $\operatorname{var}(Y)=\frac{1}{4}(1+5+2)=2$, yielding

$$
\operatorname{corr}^{2}(\mathbb{E}[N \mid \boldsymbol{T}], Y)=\frac{1}{16} \frac{\operatorname{cov}^{2}\left(T_{(2)}-T_{(1)}, T_{(2)}+T_{(1)}\right)}{2}=\frac{1}{2} .
$$

Hence, the best linear combination estimator has variance $\frac{1}{2}$.

Again, consider a parallel system with $p_{1}=p_{2}=\frac{1}{2}$, but now suppose that $n_{1}=n_{2}=2$. Now, $N$ has the distribution $3+X_{1}+I X_{2}$ where $I, X_{1}, X_{2}$ are independent, with the $X_{i}$ being geometric with parameter $\frac{1}{2}$, and $\mathbb{P}(I=1)=\frac{1}{4}=1-\mathbb{P}(I=0)$, and we easily obtain, using the conditional variance formula, that $\operatorname{var}(N)=\frac{13}{4}$. To obtain the variance of the conditional expectation estimator $\mathbb{E}[N \mid \boldsymbol{T}]=4+\frac{1}{2}\left(T_{(2)}-T_{(1)}\right)$, we use the fact that the distribution of $T_{(2)}-T_{(1)}$ is that of $Y_{1}+I Y_{2}$, where the $Y_{i}$ are exponential with rate $\frac{1}{2}, I$ is equally likely to be 0 or 1 , and $Y_{1}, Y_{2}, I$ are independent. This yields $\operatorname{var}(\mathbb{E}[N \mid \boldsymbol{T}])=\frac{7}{4}$.

\subsection{Estimating $\mathbb{E}\left[N^{2}\right]$ and $\operatorname{var}(N)$}

To estimate $\mathbb{E}\left[N^{2}\right]$, note from (1) that

$$
\operatorname{var}(N \mid \boldsymbol{T})=\sum_{j=1}^{K-1} p_{I_{j}}\left(T-T_{I_{j}}\right)+\sum_{j=K+1}^{r}\left(n_{I_{j}}-1\right) \frac{T}{T_{I_{j}}}\left(1-\frac{T}{T_{I_{j}}}\right) .
$$

Using the fact that

$$
\mathbb{E}\left[N^{2} \mid \boldsymbol{T}\right]=\mathbb{E}^{2}[N \mid \boldsymbol{T}]+\operatorname{var}(N \mid \boldsymbol{T})
$$

enables us to use (2) and (4) to obtain $\mathbb{E}\left[N^{2} \mid \boldsymbol{T}\right]$, which is an unbiased estimator of $\mathbb{E}\left[N^{2}\right]$. For a second unbiased estimator, we use (3) to obtain

$$
\mathbb{E}\left[T^{2}-T\right]=\mathbb{E}\left[N^{2}\right] .
$$

Consequently, we propose estimating $\mathbb{E}\left[N^{2}\right]$ by the best estimator of the form $\alpha \mathbb{E}\left[N^{2} \mid \boldsymbol{T}\right]+$ $(1-\alpha)\left(T^{2}-T\right)$. We can then use the estimates of $\mathbb{E}[N]$ and $\mathbb{E}\left[N^{2}\right]$ to estimate $\operatorname{var}(N)$. 


\subsection{Estimating $\mathbb{P}(N>m)$}

To estimate $\mathbb{P}(N>m)$, we propose generating the values of the $r-K$ independent binomial random variables $B_{j}(\boldsymbol{T}), j>K$, and then using the estimator

$$
\begin{aligned}
\mathbb{P}(N & \left.>m \mid \boldsymbol{T}, B_{j}(\boldsymbol{T}) \text { for } j>K\right) \\
& =\mathbb{P}\left(X(\boldsymbol{T})>m-\sum_{j=1}^{K} n_{I_{j}}-\sum_{j=K+1}^{r} B_{j}(\boldsymbol{T}) \mid \boldsymbol{T}, B_{j}(\boldsymbol{T}) \text { for } j>K\right),
\end{aligned}
$$

which, with $I=m-\sum_{j=1}^{K} n_{I_{j}}-\sum_{j=K+1}^{r} B_{j}(\boldsymbol{T})$ and $\lambda(\boldsymbol{T})=\sum_{j=1}^{K-1} p_{I_{j}}\left(T-T_{I_{j}}\right)$, can be written as

$$
\mathbb{P}\left(N>m \mid \boldsymbol{T}, B_{j}(\boldsymbol{T}) \text { for } j>K\right)= \begin{cases}1 & \text { if } I<0, \\ \sum_{i=I+1}^{\infty} \mathrm{e}^{-\lambda(\boldsymbol{T})} \frac{\lambda(\boldsymbol{T})^{i}}{i !} & \text { if } I \geq 0 .\end{cases}
$$

Remark. Because $m$ only comes into the simulation in the final computation (5), one simulation run can be used to obtain estimates of $\mathbb{P}(N>m)$ for any set of values of $m$.

\subsection{Generating $N$}

It should be noted that none of our estimates call for the generation of the random variable $N$. However, if we want to generate $N$, as we will in later sections, we could do so by first generating $\boldsymbol{T}$, then generating the (conditionally) independent random variables $X(\boldsymbol{T}), B_{j}(\boldsymbol{T})$, $j=K+1, \ldots, r$, and then setting $N=n(\boldsymbol{T})+X(\boldsymbol{T})+\sum_{j=K+1}^{r} B_{j}(\boldsymbol{T})$. Except in cases where $N$ tends to be small, this approach will usually be more efficient than the one that generates $N$ by sequentially generating the outcomes of the multinomial trials.

\section{Estimating the mean, variance, and tail distribution of the system life}

We now consider estimating the mean, variance, and tail distribution of the system life in cases where the point process is a Poisson process, a renewal process, and a Hawkes process.

\subsection{Case where the point process is a Poisson process}

Suppose that the shock counting process $\{N(t), t \geq 0\}$ is a Poisson process with rate $\lambda$. Using the fact that $\mathbb{E}[L \mid N]=N / \lambda$, we see that

$$
\mathbb{E}[L]=\frac{\mathbb{E}[N]}{\lambda} .
$$

Also, because $\operatorname{var}(L \mid N)=N / \lambda^{2}$, the conditional variance formula gives

$$
\operatorname{var}(L)=\frac{\mathbb{E}[N]+\operatorname{var}(N)}{\lambda^{2}} .
$$

Hence, we can estimate $\mathbb{E}[L]$ and $\operatorname{var}(L)$ by using the approach of Section 2 to estimate $\mathbb{E}[N]$ and $\operatorname{var}(N)$.

Now, suppose that we want to estimate $\mathbb{P}(L>t)$ for a range of values of $t$. This is accomplished by using the fact that

$$
\mathbb{P}(L>t)=\mathbb{P}(N(t)<N)=\mathbb{P}(N(t)<n(\boldsymbol{T})+X(\boldsymbol{T})+B(\boldsymbol{T})),
$$


where $N(t)$ is independent of the random variables on the right-hand side, which are given by the representation (1).

We propose to estimate $\mathbb{P}(L>t)$ by generating $\boldsymbol{T}$ and then generating the independent binomials whose sum is $B(\boldsymbol{T})$. If the generated values yield $n(\boldsymbol{T})=s, B(\boldsymbol{T})=b$, and $\sum_{j=1}^{k-1} p_{I_{j}}\left(T-T_{I_{j}}\right)=\mu$ then

$$
\mathbb{P}(N(t)<n(\boldsymbol{T})+X(\boldsymbol{T})+B(\boldsymbol{T}) \mid \boldsymbol{T}, B(\boldsymbol{T}))=\mathbb{P}(N(t)<s+b+V),
$$

where $V$ is Poisson with mean $\mu, N(t)$ is Poisson with mean $\lambda t$, and $V$ and $N(t)$ are independent. We can then use

$$
\mathbb{P}(N(t)<s+b+V)=\sum_{i=0}^{\infty} \sum_{j<s+b+i} \mathrm{e}^{-\lambda t} \frac{(\lambda t)^{j}}{j !} \mathrm{e}^{-\mu} \frac{\mu^{i}}{i !}
$$

as an estimator of $\mathbb{P}(L>t)$. Moreover, because $L$ has the same distribution as $T / \lambda$, where $T$ is defined as in Section 2, $\mathbf{1}\{T>\lambda t\}$ is also an unbiased estimator of $\mathbb{P}(L>t)$. Both $\mathbf{1}\{T>\lambda t\}$ and (6) can be obtained for multiple runs and then the best unbiased linear combination estimator can be used.

Remarks. (a) If one wants to avoid computing the right-hand side of (6), the simulation run could continue by generating the values of $N(t)$ and $V$, and then taking the conditional probabilities $\mathbb{P}(N(t)<s+b+V \mid N(t))$ and $\mathbb{P}(N(t)<s+b+V \mid V)$ as two unbiased estimators from that run. After all the simulation runs are completed, the best linear combination of the averages of the values of the two estimators over all runs can be determined.

(b) In the Poisson case considered in this section the component lifetimes are independent Erlang (i.e. gamma with parameters $(n, \alpha)$ where $n$ is a positive integer) random variables. Thus, for instance, if the structure is $k$-of- $r$ (which functions when at least $k$ of the $r$ components are working) then the system life is the $r-k+1$ order statistic of independent Erlang random variables. Thus, our approach can be compared with the use of computationally intensive recursive equations for finding the moments of such order statistics that is suggested in [1]. Interestingly, in the Poisson case we start with independent Erlang distributed component lifetimes but then analyze by interpreting it as a shock model (sort of the reverse of the usual Poissonization approach) and then employ Poissonization to obtain efficient estimators.

\subsection{Case where the point process is a renewal process}

If the shock counting process $\{N(t), t \geq 0\}$ is a renewal process with continuous interarrival distribution $F$ then $\mathbb{E}[L]$ and $\operatorname{var}(L)$ can be estimated exactly as in the Poisson process case. Namely, with $\mu$ and $\sigma^{2}$ equal to the mean and variance of an interarrival time, we note that

$$
\mathbb{E}[L]=\mu \mathbb{E}[N], \quad \operatorname{var}(L)=\mu^{2} \operatorname{var}(N)+\sigma^{2} \mathbb{E}[N],
$$

and then use the approach of Section 2 to estimate $\mathbb{E}[N]$ and $\operatorname{var}(N)$.

Suppose now that we want to estimate $\mathbb{P}(L>t)$. We can obtain an unbiased estimator by first using the method of Section 2.4 to generate a random variable $N$. If the generated value is $N=n$ then $\mathbb{P}\left(X_{1}+\cdots+X_{n}>t\right)$ is an unbiased estimator of $\mathbb{P}(L>t)$, where, throughout this section, $X_{1}, X_{2}, \ldots$, are independent random variables with distribution $F, S_{j}=\sum_{i=1}^{j} X_{i}$, and $M_{j}=\max _{i \leq j} X_{i}$. Because $\mathbb{P}\left(X_{1}+\cdots+X_{n}>t\right)$ is usually not easily computed, we propose to estimate it by using the modification (given in [9]) of the Asmussen-Kroese (AK) simulation approach for estimating a convolution (see [3]). We now outline this approach for estimating $\mathbb{P}\left(X_{1}+\cdots+X_{n}>t\right)$. 


\subsubsection{Modified AK approach to estimate $\mathbb{P}\left(S_{n}>t\right)$.}

- Noting that $P_{n} \equiv \mathbb{P}\left(S_{n}>t\right)=n \mathbb{P}\left(S_{n}>t, M_{n}=X_{n}\right)$, the AK estimator of $P_{n}$ is

$$
\mathcal{E}_{\mathrm{AK}}=n \mathbb{P}\left(S_{n}>t, M_{n}=X_{n} \mid X_{1}, \ldots, X_{n-1}\right)=n \bar{F}\left(\min \left(M_{n-1}, t-S_{n-1}\right)\right),
$$

where $\bar{F}=1-F$.

- Let $R=\min \left\{n-1, \min \left\{j \geq 1: M_{j}+S_{j}>t\right\}\right\}$. Conditioning on $R, X_{1}, \ldots, X_{R}$ gives the improved AK estimator:

$$
\begin{aligned}
\mathcal{E} & =n \mathbb{P}\left(S_{n}>t, X_{n}=M_{n} \mid R, X_{1}, \ldots, X_{R}\right) \\
& = \begin{cases}\frac{n}{n-R}\left(1-F^{n-R}\left(M_{R}\right)\right) & \text { if } R<n-1, \\
\mathcal{E}_{\mathrm{AK}} & \text { if } R=n-1 .\end{cases}
\end{aligned}
$$

When $R<n-1$, the preceding equation follows because $\mathbb{P}\left(S_{n}>t, X_{n}=M_{n} \mid R\right.$, $\left.X_{1}, \ldots, X_{R}\right)$ is the probability of the joint event that $X_{n}$ is both the largest of the values $X_{R+1}, \ldots, X_{n}$ and is itself greater than $M_{R}$; and this is equal to the probability of the joint event that at least one of $X_{R+1}, \ldots, X_{n}$ exceeds $M_{R}$ and that $X_{n}$ is the largest of these $n-R$ values.

We now present our approach for using simulation to estimate $\mathbb{P}(L>t)$. Fix a value $k$ and use the approach of Section 2.4 to generate $k$ independent random variables having the distribution of $N$. Let $m_{1} \leq m_{2} \leq \cdots \leq m_{k}$ be the ordered sequence of these $k$ generated values. To simultaneously estimate $\mathbb{P}\left(S_{m_{i}}>t\right)$ for each $i=1, \ldots, k$, generate $X_{1}, \ldots, X_{m_{k}}$ and use these data to estimate $\mathbb{P}\left(S_{m_{i}}>t\right)$ via the modified AK approach. That is, with $R=\min \left\{m_{k}-1, \min \left\{j \geq 1: M_{j}+S_{j}>t\right\}\right\}$, the estimate of $\mathbb{P}\left(S_{m_{i}}>t\right)$ is

$$
\mathcal{E}_{m_{i}}= \begin{cases}\frac{m_{i}}{m_{i}-R}\left(1-F^{m_{i}-R}\left(M_{R}\right)\right) & \text { if } R<m_{i}-1, \\ m_{i} \bar{F}\left(\min \left(M_{m_{i}-1}, t-S_{m_{i}-1}\right)\right) & \text { if } R \geq m_{i}-1 .\end{cases}
$$

The quantity $1 / k \sum_{i=1}^{k} \varepsilon_{m_{i}}$ is the estimate of $\mathbb{P}(L>t)$ from this run. These runs can be then repeated in the usual simulation manner to obtain a final estimate (equal to the average of the estimates over all runs.)

Remarks. An appropriate choice of $k$ can be learned only from the simulation. The reason for having $k>1$ is that doing so yields, in each run, $k$ unbiased estimates, with a computational effort that increases concavely in $k$. Although these $k$ estimators are dependent (and almost certainly positively correlated), the following lemma shows that the run estimator, equal to their average, has a variance that decreases in $k$.

Lemma 1. If $W_{1}, W_{2} \ldots$, is a sequence of exchangeable random variables, $\operatorname{var}\left(\sum_{i=1}^{k} W_{i} / k\right)$ decreases in $k$.

Proof. Let $\sigma^{2}=\operatorname{var}\left(W_{1}\right)$ and $c=\operatorname{cov}\left(W_{1}, W_{2}\right)$. Using the formula for the variance of a sum, we need to show that

$$
\frac{(k+1) \sigma^{2}+(k+1) k c}{(k+1)^{2}} \leq \frac{k \sigma^{2}+k(k-1) c}{k^{2}},
$$

which reduces to $c \leq \sigma^{2}$, and this is the case since $c / \sigma^{2}=\operatorname{corr}\left(W_{1}, W_{2}\right)$. 


\subsection{Case where the point process is a Hawkes process}

A point process $\{N(t), t \geq 0\}$ is said to be a marked Hawkes process (see [4]) if its conditional intensity function is given by

$$
\Lambda(t)=\lambda+\sum_{i=1}^{N(t)} M_{i} \mathrm{e}^{-\alpha\left(t-S_{i}\right)},
$$

where $S_{i}, i \geq 1$, are the successive event times of the point process, $\lambda$ and $\alpha$ are specified positive constants, and the sequence of marks $M_{i}, i \geq 1$, are independent and identically distributed positive random variables having a specified distribution $G$. In addition, $M_{i}$ is independent of $S_{1}, \ldots, S_{i}$. The idea is that event $i$ has a mark $M_{i}$ attached to it, with the effect being that the current value of the intensity function increases by $M_{i}$ when event $i$ occurs; moreover, the increment of the intensity function beyond its base value $\lambda$ decreases between events at an exponential rate $\alpha$. Thus, for instance,

$\mathbb{P}(N(s+t+h)-N(s+t)=1 \mid \Lambda(t)=\lambda+v, N(s+t)-N(t)=0)=\left(\lambda+v \mathrm{e}^{-\alpha s}\right) h+o(h)$.

It is easy to simulate a Hawkes process by simulating its successive event times $S_{i}, i \geq 1$. The following algorithm simulates the first $n$ event times. The value $V_{i}$ is such that $\lambda+V_{i}$ is the intensity rate immediately after event $i$. (If we wanted to simulate up to a fixed time $t$, just replace 'If $J=n$ stop' in step 4 with 'if $S_{J}>t$ stop'. The final value of $J$ would equal $N(t)+1$.

Step 1. Let $S=0, V=0$, and $J=1$.

Step 2. Generate $X$, having hazard rate function $h(s)=\lambda+V \mathrm{e}^{-\alpha s}, s>0$.

Step 3. Let $S_{J}=S+X$.

Step 4. If $J=n$ stop.

Step 5. Generate $M$ having distribution function $G$.

Step 6. Let $V_{J}=V \mathrm{e}^{-\alpha X}+M$.

Step 7. Let $V=V_{J}, S=S_{J}$, and $J=J+1$.

Step 8. Go to step 2.

Remark. Step 2 generates a random variable $X$ with hazard rate function $h(s)=\lambda+V \mathrm{e}^{-\alpha s}$. As $X$ has distribution function $F(t)=1-\exp \left\{-\int_{0}^{t} h(s) \mathrm{d} s\right\}=1-\exp \left\{-\lambda t-V\left(1-\mathrm{e}^{-\alpha t}\right) / \alpha\right\}$, it could be generated by the inverse transform method by generating a uniform $(0,1)$ random variable $U$ and then numerically solving

$$
-\log (1-U)=\lambda X+V \frac{1-\mathrm{e}^{-\alpha X}}{\alpha} .
$$

Because the right-hand side of the preceding equation is increasing in $X$, the computations are not onerous. Another approach would be to generate the time of the first event of a nonstationary Poisson process with intensity function $\lambda+V \mathrm{e}^{-\alpha s}$, by using the thinning method on a Poisson process with rate $\lambda+V$ for example, and then set $X$ equal to the generated value. 
Suppose now that the shock counting process is a Hawkes process, and that we want to estimate $\mathbb{E}[L], \mathbb{E}\left[L^{2}\right]$, and $\mathbb{P}(L>t)$. To do so, fix a value $k$. The first run of the simulation begins by using the method of Section 2.4 to generate $k$ independent replicas of $N$, say $N_{1}, \ldots, N_{k}$. Let their generated values be $m_{i}, i=1, \ldots, k$, and set $m=\max _{i} m_{i}$. Now generate the first $m$ event times of the Hawkes process: $S_{1}, \ldots, S_{m}$.

The values of the unbiased estimators of $\mathbb{E}[L]$ and $\mathbb{E}\left[L^{2}\right]$ from this run are, respectively, $\sum_{i=1}^{k} S_{m_{i}} / k$ and $\sum_{i=1}^{k} S_{m_{i}}^{2} / k$. With $\mathbb{E}[M]$ being the expected value of a mark, these estimators can be improved by using $\sum_{i=1}^{\max _{i} N_{i}}\left(M_{i}-\mathbb{E}[M]\right)$ as a control variable whose mean is 0 since $\max _{i} N_{i}$ is independent of the sequence of marks. Because the control variable will be large (small) when marks are higher (lower) than average it should be negatively correlated with system lifetime.

To estimate $\mathbb{P}(L>t)$, we first estimate $\mathbb{P}\left(S_{m_{i}}>t\right)$ by the estimator

$$
\mathcal{E}_{m_{i}} \equiv \mathbb{P}\left(S_{m_{i}}>t \mid S_{m_{i}-1}, V_{m_{i}-1}\right)
$$

given by

$$
\mathcal{E}_{m_{i}}= \begin{cases}\exp \left\{-\int_{0}^{t-S_{m_{i}-1}}\left(\lambda+V_{m_{i}-1} \mathrm{e}^{-\alpha s}\right) \mathrm{d} s\right\} & \text { if } S_{m_{i}-1}<t, \\ 1 & \text { if } S_{m_{i}-1} \geq t,\end{cases}
$$

and then take $\mathcal{E}=\sum_{i=1}^{k} \varepsilon_{m_{i}} / k$ as the estimate of $\mathbb{P}(L>t)$ from the run. In addition, the estimator can be improved by using $\sum_{i=1}^{N(t)}\left(M_{i}-\mathbb{E}[M]\right)$ as a control variable. Because the event $N(t)=j$ is independent of all marks observed after time $t$, it follows that it is independent of $M_{j+1}, M_{j+2}, \ldots$ and so is a stopping time for the sequence $M_{1}, M_{2}, \ldots$ Hence, by Wald's equation, $\mathbb{E}\left[\sum_{i=1}^{N(t)}\left(M_{i}-\mathbb{E}[M]\right)\right]=0$.

\section{Concluding remarks}

There are many interesting generalizations of our model, with our techniques being directly applicable in some but not in others. For instance, one generalization might suppose that the number of type- $i$ shocks that it takes until component $i$ fails is a random variable $W_{i}$, with $W_{1}, \ldots, W_{r}$ having an arbitrary joint distribution. For this model, we could first generate the values of $W_{1}, \ldots, W_{r}$, and then utilize our techniques (possibly also using antithetic variables or control variables as related to the generated $W_{1}, \ldots, W_{r}$.) Other generalizations might be to suppose that a shock could affect multiple components, or that there is some specified decreasing function $h$ such that the system performs at a level $h\left(x_{1}, \ldots, x_{r}\right)$ if there have been a total of $x_{i}$ type- $i$ shocks, for each $i=1, \ldots, r$, with the system failing when this level falls below some specified value. For these latter generalizations, new techniques for simulation efficiency would be needed.

Also, in this paper we have not addressed rare event simulation that arises in cases where the probabilities of interest are quite small.

\section{References}

[1] Abdelkader, Y. H. (2004). Computing the moments of order statistics from nonidentical distributed Erlang variables. Statist. Papers 45, 563-570.

[2] Aldous, D. (1989). Probability Approximations via the Poisson Clumping Heuristic. Springer, New York.

[3] Asmussen, S. And Kroese, D. P. (2006). Improved algorithms for rare event simulation with heavy tails. Adv. Appl. Prob. 38, 545-558. 
[4] Daley, D. J. And Vere-Jones, D. (2003). An Introduction to the Theory of Point Processes, Vol 1, 2nd edn. Springer, New York.

[5] Esary, J. D., Marshall, A. W. and Proschan, F. (1973). Shock models and wear processes. Ann. Prob. 1, 627-650.

[6] Holst, L. (1986). On birthday, collectors', occupancy and other classical urn problems. Internat. Statist. Rev. 54, 15-27.

[7] Ross, S. M. (2013). Simulation, 5th edn. Academic Press, San Diego.

[8] Sobel, M. and Ebneshahrashoob, M. (1992). Quota sampling for multinomial via Dirichlet. J. Statist. Planning Infer. 33, 157-164.

[9] Ghamami, S. and Ross, S. M. (2012). Improving the Asmussen-Kroese type simulation estimators. J. Appl. Prob. 49, 1188-1193. 